\title{
Quantitative Analysis of Magnetic Excitations in Landau Flux-Closure Structures Using Synchrotron-Radiation Microscopy
}

\author{
J. Raabe, ${ }^{1, *}$ C. Quitmann, ${ }^{1}$ C. H. Back, ${ }^{2}$ F. Nolting, ${ }^{1}$ S. Johnson, ${ }^{1}$ and C. Buehler ${ }^{1}$ \\ ${ }^{1}$ Paul Scherrer Institut, CH-5232 Villigen - PSI, Switzerland \\ ${ }^{2}$ Institut für Experimentelle Physik, Universität Regensburg, D-93040 Regensburg, Germany
}

(Received 16 December 2004; published 2 June 2005)

\begin{abstract}
We have measured the excitation spectrum of six micron magnetic squares using x-ray magnetic circular dichroism. We observe all three excitations expected in a Landau flux-closure pattern. High temporal and spatial resolution allows quantitative analysis of the excitations. A short magnetic in plane pulse excites the magnetic element and we observe precessional motion of the magnetization within the domains as well as a domain wall mode and vortex motion. The vortex moves perpendicular to the excitation field and relaxes without showing a circulating orbit.
\end{abstract}

The arrangement of magnetic moments in micro- and nanostructures and their excitations have recently attracted considerable attention. Micromagnetic calculations are now able to predict the magnetic equilibrium state precisely and have been verified experimentally. The dynamics of the magnetization in such small elements is much more challenging. Micron or submicron magnetic particles can minimize their stray field energy by an inhomogeneous magnetization. A uniform square, for example, assumes a Landau flux-closure pattern [1] consisting of (i) domains with a homogenous magnetization, (ii) domain walls separating them, and (iii) a vortex formed at the intersection of domain walls. Each of these substructures shows excitations characteristic of the local total internal field.

Recent experimental and theoretical work has yielded a significant understanding of homogenously magnetized objects and the simplest inhomogeneous object, a disk containing a vortex in the center [2-8]. However, a disk does not contain domain walls. These are found in squares, but little is known about their excitation spectrum. Park et al. have studied the domain wall mode and the fundamental domain mode. Choe et al. [9] have observed a circulating orbit, a so-called gyrotropic motion, of the vortex which was predicted theoretically [10] and was previously studied using resonant excitations in garnets [11]. The influence of domain walls on magnetization reversal was studied by Kuch et al. [12].

The time dependent magnetization is described by the phenomenological Landau-Lifshitz-Gilbert equation

$$
\frac{d}{d t} \vec{M}=-\gamma_{0} \vec{M} \times \vec{H}_{\mathrm{eff}}+\frac{\alpha}{M}\left(\vec{M} \times \frac{d}{d t} \vec{M}\right) .
$$

The first term describes the precession of the magnetization $\vec{M}$ about the total effective field $\vec{H}_{\text {eff }}$. The second term describes the relaxation back into the equilibrium state using the dimensionless damping parameter $\alpha$. For small amplitudes, the precessional frequency is given by $\omega_{0}=\gamma_{0} H_{\text {eff }}$, where $\gamma_{0}$ is the gyromagnetic ratio and $\vec{H}_{\text {eff }}$ is the partial derivative of the total energy density with respect to the magnetization, $\delta \varepsilon_{\text {tot }} / \delta \vec{M}$.

The magnetization can be excited by a short magnetic field pulse oriented either within the plane $[4,9]$ or perpendicular to it $[13,14]$. Depending on the amplitude three cases can be distinguished. Weak pulses cause small amplitude excitations, strong pulses result in larger amplitude nonlinear excitations, and even higher pulses cause switching, the irreversible rearrangement of domains.

In Landau flux-closure structures the three substructures (domains, domain walls, vortex) differ in their energy densities $\varepsilon_{\text {tot }}$ leading to differences in the local $\vec{H}_{\text {eff }}$ and to three distinct frequencies or time scales. Within the domain the magnetization is constant and $\varepsilon_{\text {tot }}$ is dominated by the magnetostatic energy. In the vortex core, where the magnetization rotates from in plane to out of plane and back within $\approx 50 \mathrm{~nm}[15], \varepsilon_{\text {tot }}$ is dominated by the exchange energy. The domain walls are an intermediate case with contributions from both dipole and exchange energy.

In this Letter we analyze quantitatively the complete excitation spectrum in micron sized permalloy squares. The time dependent spatial distribution of the magnetization $\vec{M}(\vec{r}, t)$ was measured using a photoemission electron microscope (PEEM) following strong in plane excitations. The samples are permalloy $\left(\mathrm{Ni}_{81} \mathrm{Fe}_{19}\right)$ squares of various orientation with $6 \mu \mathrm{m}$ length and $30 \mathrm{~nm}$ thickness, placed on a common coplanar waveguide (gold: $10 \mu \mathrm{m}$ width, $200 \mathrm{~nm}$ thickness). They are imaged using the PEEM at the Surface/Interface Microscopy (SIM) beam line of the Swiss Light Source (SLS) [16]. The PEEM records the $\mathrm{x}$-ray absorption coefficient with a spatial resolution of $\approx 100 \mathrm{~nm}$. X-ray magnetic circular dichroic (XMCD) images are obtained by taking two images at the Fe- $L_{\text {III }}$ absorption edge differing only in the direction of the circular polarization $(C+$ and $C-)$. Taking their ratio eliminates topographic and chemical contrast [17]. The intensity $I(\vec{r})$ is then proportional to the scalar product $\vec{M}(\vec{r}) \cdot \vec{P}$. Since the $\mathrm{x}$ rays are incident at $84^{\circ}$ to the surface 
normal, the experiment is mainly sensitive to the in plane component of $\vec{M}$ along $\vec{P}, M_{y}(\vec{r})$.

Time resolved experiments are performed using a stroboscopic pump-probe technique. A pulsed laser $\left(t_{\mathrm{FWHM}}<\right.$ $15 \mathrm{ps}$ ) illuminates a photodiode launching a current pulse in the coplanar waveguide. This creates a magnetic field pulse exciting the magnetization. The field amplitude is $H_{p} \approx 60 \mathrm{Oe}$ and rise and fall times are 150 and $300 \mathrm{ps,}$ respectively. The sample is probed using an x-ray pulse produced by an isolated electron bunch $\left(t_{\mathrm{FWHM}} \approx 70 \mathrm{ps}\right)$ located in a 180 ns gap of the filling pattern of the SLS storage ring [18]. All other X-ray pulses are suppressed by gating the detector. Pump and probe pulses are synchronized with a variable electronic delay $\Delta t$. The sample is excited every $16 \mathrm{~ns}$ and is probed every $1.04 \mu \mathrm{s}$. To acquire an image we typically integrate the signal for $300 \mathrm{~s}$, thus averaging over $3 \times 10^{8}$ pump-probe cycles.

The time evolution of the magnetization in a permalloy square showing a Landau flux-closure pattern is shown in Fig. 1 and is available as a movie [19]. The square is oriented parallel to the polarization $\vec{P}$ and the exciting magnetic field pulse $\vec{H}_{p}$. Shown are five snapshots representing different steps in the excitation process. The first row shows $M_{y}(\vec{r}, \Delta t)$, the center row shows difference images, obtained by subtracting the equilibrium state $(\Delta t=0)$ [20] from the images. The third row shows the schematic arrangement with arrows depicting the magne-
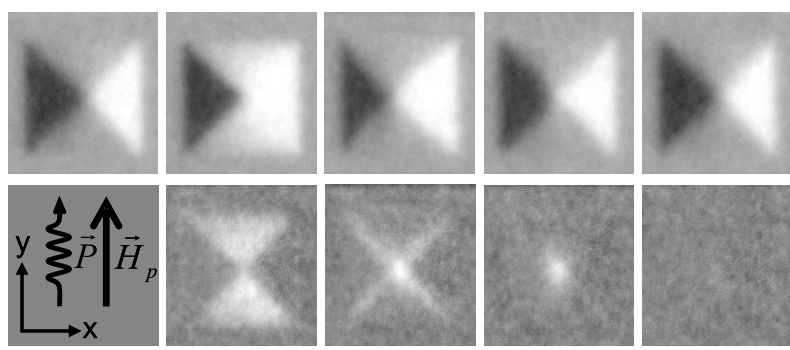

$1550 \mathrm{ps}$

2550 ps
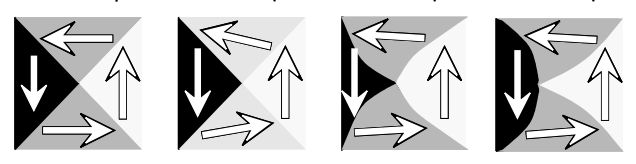

14300 ps
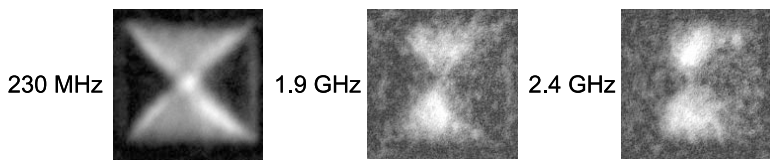

FIG. 1. Selected XMCD images showing the time evolution of the $y$ component of the magnetization (bright areas are magnetized upward, dark areas downward) in $6 \mu \mathrm{m}$ permalloy squares (first row). Difference images obtained by subtracting the equilibrium state $(\Delta t=0)$ from each image (second row). The orientation of the exciting field pulse and the polarization $\vec{P}$ are sketched in the leftmost image. Sketches of the prominent features for different delay times (third row). In the fourth row we show images of the Fourier amplitudes obtained by Fourier transforming the image sequence. tization direction and gray levels corresponding to the image intensity. At $\Delta t=0$ we image the system before the excitation, thus observing the Landau flux-closure equilibrium state. The domains oriented parallel and antiparallel to $\vec{P}$ appear white and black, while the two oriented perpendicular to $\vec{P}$ both appear gray. Straight domain walls separate the triangular domains and the vortex is in the center of the square [21]. At $\Delta t=300 \mathrm{ps}$ the intensity has increased in the two domains with $\vec{M} \perp \vec{H}_{p}$, while the others show no change. The two bright triangles in the difference image substantiate this. For somewhat longer delay times $(\Delta t=1550 \mathrm{ps})$ the intensity of domains with $\vec{M} \perp \vec{H}_{p}$ has decayed back to the equilibrium state value, while the domain walls and the vortex now show pronounced changes. The vortex has moved to the left, perpendicular to the applied field pulse $\vec{H}_{p}$. This is in contrast to a recent paper by Choe et al. [9] where an initial motion parallel to $\vec{H}_{p}$ was observed in a similar experiment. We will address this discrepancy when discussing Fig. 2. At $\Delta t=1550 \mathrm{ps}$ the domain walls are no longer straight lines. They bulge to the left, the direction of the vortex motion. The difference image shows a bright spot in the center and a bright cross, corresponding to the vortex motion and the domain wall bulging, respectively. The next step is depicted for $\Delta t=2550 \mathrm{ps}$. The bulging of the domain wall has reversed (see first row) indicating that they have undergone a complete oscillation period. The vortex is still displaced perpendicular to $\vec{H}_{p}$ as evidenced by the bright spot in the difference image. The domain wall bulging is less visible in the difference image, as all images are scaled to the same contrast. Apparently, the speed of the domain walls exceeds that of the vortex. For long delay times the system relaxes back to the equilibrium state as seen for $\Delta t=14300 \mathrm{ps}$. Here the image is indistinguishable from the equilibrium state as confirmed by the difference image. Since the excitation pulses are spaced by
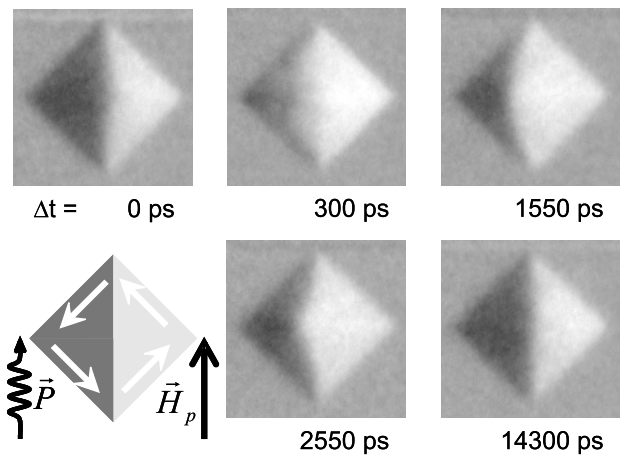

FIG. 2. Series of XMCD images showing the time evolution of the magnetic contrast in $6 \mu \mathrm{m}$ permalloy squares for the same field pulse excitation as in Fig. 1. Here, the squares are rotated by $45^{\circ}$ with respect to the excitation $\vec{H}_{p}$ and the polarization P. The $\mathrm{XMCD}$ contrast and excitation geometry are schematically shown in the lower left. 
$16 \mathrm{~ns}$, the system relaxes back to the equilibrium state before being reexcited in this stroboscopic experiment.

The different processes and time scales observed in the excitation are shown schematically in the third row. In the following we treat the processes separately for pedagogic reasons. This neglects their coupling but allows determination of frequencies, amplitudes, and decay rates for each of the three processes. The equilibrium state is sketched in the third row (left) of Fig. 1. During the excitation the magnetic field pulse $\vec{H}_{p}$ tips the effective field causing a torque $\vec{T}=-\gamma_{0} \vec{M} \times \vec{H}_{\text {eff }}$. For the squares oriented parallel to $\vec{H}_{p}$ the torque is zero in the black and the white domains. In the gray domains the torque tilts the magnetization away from its equilibrium state. Once the field pulse has decayed, the magnetization relaxes back, performing a damped precessional motion. The precession frequency is mainly determined by the dipolar energy thus depending on the shape, size, and on material parameters $\left(M_{s}\right.$ and $\left.\gamma_{0}\right)$. It is the highest frequency observed in our experiment as expected from the energetics of the Landau flux-closure pattern. After half a precession period $(\Delta t=300 \mathrm{ps})$ the magnetization has a maximum component along $\vec{P}$ thus showing maximum intensity. For longer delay times domain wall excitations and vortex displacement dominate. The oscillation period of the domain wall is longer than for the domain precession because of the altered effective field $\vec{H}_{\text {eff }}$ [4]. The excitation of the domain wall is an oscillation [19], analogous to an excited string, but complicated by the fact that one of the end points - the vortex - is moving. The vortex motion sets the slowest time scale.

The modes and their frequencies can be determined using a Fourier transformation of the image series $[4,5,14]$. Images of the spatially resolved Fourier amplitude for three frequencies are shown in the third row of Fig. 1. At $230 \mathrm{MHz}$ the intensity is highest along the domain walls, while at 1.9 and $2.4 \mathrm{GHz}$ the intensity is highest within the domains. These frequencies correspond to a domain wall mode and two normal modes within the domain. The phase images of the 1.9 and $2.4 \mathrm{GHz}$ modes show a uniform phase within the two domains but $180^{\circ}$ phase shift between the two modes.

To determine the initial direction of vortex motion and to clarify the discrepancy with the results of [9] we investigated squares of equal size, but rotated by $45^{\circ}$ with respect to both $\vec{P}$ and $\vec{H}_{p}$. They are placed on the same waveguide as those in Fig. 1 and are measured in the same experiment, thus experiencing the same excitation. In these rotated squares the excitation is topologically different. All domains have a component of $\vec{M}$ perpendicular to $\vec{H}_{p}$ and experience a torque of equal magnitude. An image series is shown in Fig. 2 [19]. The equilibrium state $(\Delta t=0)$ [20] shows two triangles with different gray levels indicative of the four domains in a Landau pattern.

At $\Delta t=300 \mathrm{ps}$ the intensity has increased in all domains, consistent with equal precessional motion caused by the initial torque. For $\Delta t=1550 \mathrm{ps}$ we observe a large vortex displacement which is again perpendicular to $\vec{H}_{p}$ confirming our observation of an initial vortex motion perpendicular to the applied field pulse. The bulging of the domain walls is even larger than in Fig. 1. At $\Delta t=$ $2550 \mathrm{ps}$ the domain walls have bulged back while the vortex has not yet relaxed to the center of the square. For long delays ( $>\approx 14300 \mathrm{ps}$ ) the system has returned to the Landau pattern ensuring that it starts from the equilibrium state when being reexcited after 16000 ps.

To determine the frequencies and amplitudes of the data shown in Fig. 1 we analyze $M_{y}(\Delta t)$ by averaging over the boxes shown in the inset of Fig. 3. The white domain $(\vec{M} \|$ $\vec{P}$ ) shows only a small variation of $M_{y}(\Delta t)$, see Fig. 3(a). This is to be expected since there is no initial torque acting on it. In contrast, the gray domains $(\vec{M} \perp \vec{P})$ show four pronounced peaks at $\Delta t=300,6661166$, and $2233 \mathrm{ps}$, indicative of damped oscillations. To analyze the data we decompose it into an oscillatory part and a slowly varying background, determined using a running average of $\pm 200 \mathrm{ps}$ around the data point. It is shown by the dashed line in Fig. 3(a). The oscillatory part can be described by the sum of two damped oscillators with a phase shift of $\pi$ and a single decay time $\tau$ [Fig. 3(b)]:

$$
M_{y}(\Delta t)=M_{s}\left[A_{1} \sin \left(\omega_{1} \Delta t\right)+A_{2} \sin \left(\omega_{2} \Delta t+\pi\right)\right] e^{-\Delta t / \tau}
$$

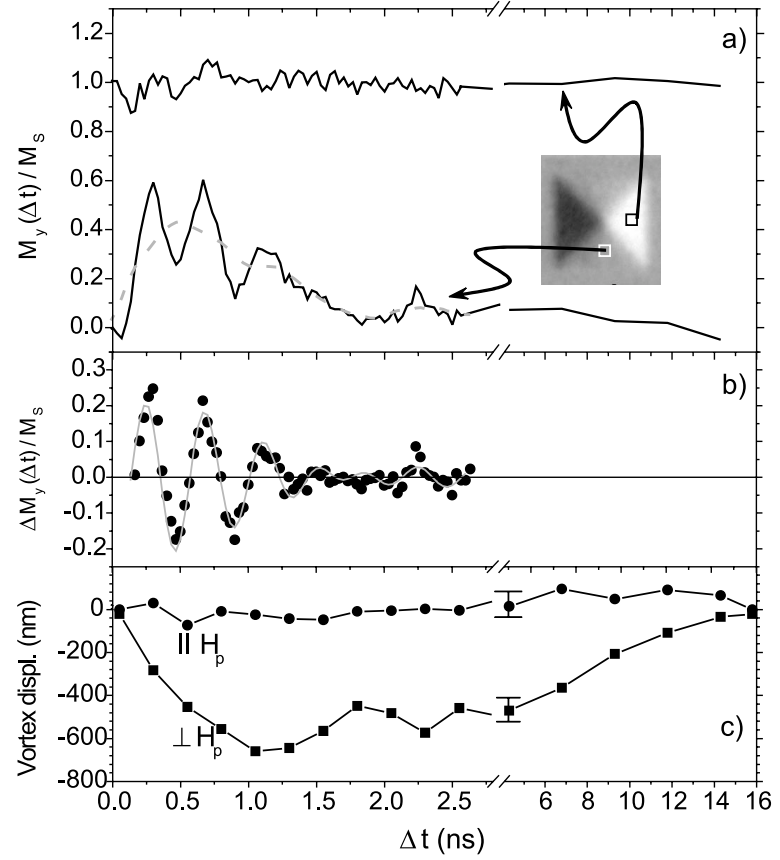

FIG. 3. Quantitative analysis of the time dependent magnetization extracted from the image series shown in Fig. 1: (a) time evolution of $M_{y}$ averaged over the boxes shown in the inset; (b) same data as in the lower half of (a) but after subtracting the running average together with a fit to the data as described in the text; (c) vortex displacement parallel and perpendicular to $\vec{H}_{p}$. 
This is in agreement with the two precessional frequencies observed within the domain (Fig. 1). The solid line shows a fit for two oscillators with $\omega_{1} /(2 \pi)=1.9 \mathrm{GHz}$ and $\omega_{2} /(2 \pi)=2.5 \mathrm{GHz}$, and a decay time $\tau=0.63 \mathrm{~ns}$.

Ivanov and Zaspel [6] have shown that for ferromagnetic disks in the vortex state the normal mode frequencies split up into doublets due to magnon scattering at the vortex core. This splitting may be the effect observed here for the first time experimentally, albeit for a Landau pattern where a detailed theoretical analysis is still missing.

The frequency of the domain wall excitation itself can be determined from the wall bulging. We observe maximum inward and outward bulging for $\Delta t=600$ and $2300 \mathrm{ps}$ [19], corresponding to $\nu_{\text {wall }} \approx 290 \mathrm{MHz}$ in good agreement with the Fourier analysis (Fig. 1).

The vortex position is measured by comparing the intensity in four boxes, similar to a quadrant detector. The dominant vortex displacement is perpendicular to $\vec{H}_{p}$, while the displacement parallel to $\vec{H}_{p}$ is smaller by at least an order of magnitude, see Fig. 3(c). Within experimental error we see no gyrotropic vortex motion. This is in contrast to [9], but in line with micromagnetic simulations for strong field pulses [22].

One significant difference between the two experiments seems to be the excitation frequency. We excite the system every $16 \mathrm{~ns}$, after it has already decayed to the equilibrium state (Fig. 1, second row). For [9] the system is already reexcited after $8 \mathrm{~ns}$. At this time the vortex still moves with about $100 \mathrm{~m} / \mathrm{s}$ and the system has not yet reached the equilibrium state. Thus the excitation seems to be resonant, which would allow observation of modes having very small amplitudes when using pulsed excitations. Resonant excitation is known to produce gyrotropic vortex motion as shown by Argyle et al. [11] and Puzic et al. [23].

In our experiment a quantitative analysis of the vortex motion gives a maximum displacement of $750 \mathrm{~nm}$ which is reached after $1050 \mathrm{ps}$, corresponding to a velocity of $v_{\text {vortex }} \approx 700 \mathrm{~m} / \mathrm{s}$. The vortex displacement persists long after the field pulse is gone $(\approx 450 \mathrm{ps})$. It can therefore not be caused by the field pulse itself, but must be driven by the energy deposited into the domains and domain walls. A similar analysis was performed for the rotated squares of Fig. 2 (not shown). Again, the vortex motion is predominately perpendicular to $\vec{H}_{p}$ and we find no gyrotropic oscillation. The maximum vortex displacement of $1250 \mathrm{~nm}$ is reached after $600 \mathrm{ps}$ resulting in a vortex speed of $v_{\text {vortex }} \approx 2000 \mathrm{~m} / \mathrm{s}$. The higher absolute vortex displacement and velocity both indicate higher driving forces.

In conclusion, we have observed all three topological excitations expected in a Landau flux-closure pattern. The vortex motion is perpendicular to the exciting field as confirmed by squares of various orientations. Quantitative analysis shows a damped precessional oscillation within the domains, wall excitations reminiscent of an oscillating string, and strongly damped motion of the vortex core. The precessional motion of the magnetization within the domains has been shown for the first time using a high resolution magnetic microscope $(\Delta x<100 \mathrm{~nm})$, thus opening the possibility to access magnetization dynamics for submicron elements with high spatial resolution.

We gratefully acknowledge support from D. Weiss (Univ. of Regensburg) for use of the cleanroom and G. Ingold (Paul Scherrer Institut) for his work on the polarized undulators. Part of this work was performed at the Swiss Light Source, Paul Scherrer Institut, Villigen, Switzerland.

*Electronic address: joerg.raabe@psi.ch

[1] L. D. Landau and E. M. Lifshitz, Phys. Z. Sowjetunion 8, 153 (1935).

[2] W. K. Hiebert, A. Stankiewicz, and M. R. Freeman, Phys. Rev. Lett. 79, 1134 (1997).

[3] Y. Acremann et al., Science 290, 492 (2000).

[4] J. P. Park et al., Phys. Rev. B 67, R020403 (2003).

[5] M. Buess et al., Phys. Rev. Lett. 93, 077207 (2004).

[6] B. A. Ivanov and C. E. Zaspel, Appl. Phys. Lett. 81, 1261 (2002); B. A. Ivanov and C. E. Zaspel, Phys. Rev. Lett. 94, 027205 (2005).

[7] V. Novosad et al., Phys. Rev. B 66, 052407 (2002).

[8] L. Giovannini et al., Phys. Rev. B 70, 172404 (2004).

[9] S.-B. Choe et al., Science 304, 420 (2004).

[10] A. A. Thiele, Phys. Rev. Lett. 30, 230 (1973).

[11] B.E. Argyle, E. Terrenzio, and J. C. Slonczewski, Phys. Rev. Lett. 53, 190 (1984).

[12] W. Kuch et al., Appl. Phys. Lett. 85, 440 (2004).

[13] H. Stoll et al., Appl. Phys. Lett. 84, 3328 (2004).

[14] K. Perzlmaier et al., Phys. Rev. Lett. 94, 057202 (2005).

[15] A. Wachowiak et al., Science 298, 577 (2002).

[16] C. Quitmann et al., Surf. Sci. 480, 173 (2001).

[17] Magnetic Microscopy of Nanostructures Series: NanoScience and Technology, edited by H. Hopster and H. P. Oepen (Springer, New York, 2005).

[18] B. Kalantari et al., Proceedings of EPAC 2004, Lucerne, Switzerland, http://accelconf.web.cern.ch/accelconf/e04/ PAPERS/MOPKF006.PDF.

[19] See EPAPS Document No. E-PRLTAO-94-008523 for a description of the experimental setup and movies of the data shown in Figs. 1 and 2. A direct link to this document may be found in the online article's HTML reference section. The document may also be reached via the EPAPS homepage (http://www.aip.org/pubservs/ epaps.html) or from ftp.aip.org in the directory /epaps/. See the EPAPS homepage for more information.

[20] $\Delta t=0$ is defined as the onset of the excitation pulse which can be determined using the intensity change caused by the applied current pulse.

[21] The domain walls and the vortex are smaller than the resolution of the instrument, but show up as a line or a point separating the domains with uniform intensity.

[22] R. Hertel and J. Kirschner, J. Magn. Magn. Mater. 270, 364 (2004).

[23] A. Puzic et al., J. Appl. Phys. 97, 10 E704 (2005). 